\title{
The Surgical Treatment for Recurrent Liposarcoma of the Hypopharynx in a Pregnant Woman
}

\author{
Takezumi Nishihoria ${ }^{a}$ Mitsuhiro Aokia, d, Kaori Aoki ${ }^{a}$, Nansei Yamada ${ }^{a}$, Kuze Bunya ${ }^{a}$, \\ Keisuke Mizuta a, Yatsuji Ito ${ }^{a}$, Nobuyuki Hirose ${ }^{b}$, Hiroki Kato ${ }^{c}$
}

\begin{abstract}
Primary liposarcoma in the pyriform sinus of the hypopharynx is extremely rare and shows a distinct male preponderance. The complete resection has been a choice of the treatment, however the rate of recurrence is relatively high. This report presents a pregnant case with well-differentiated liposarcoma of the hypopharynx. The recurrent tumor involving the pyriform sinus and aryepiglottic folds was removed by an intraoral approach using direct suspension microlaryngoscopy and a Holmium-YAG laser. The tumor size was 30 $\mathrm{mm} \times 15 \mathrm{~mm}$ x $10 \mathrm{~mm}$. An additional systemic examination showed no distant metastasis. The patient delivered a healthy female baby without any trouble. The well-differentiated type of liposarcoma frequently shows local recurrence; however it rarely shows lymph node or distant metastasis. A complete function preserving excision such as intraoral approach may therefore appear to be an adequate management plan, however careful follow-up of these patients at regular intervals is also imperative for detecting of recurrence.
\end{abstract}

Keywords: Liposarcoma; Hypopharynx; Female; Pregnant

\section{Introduction}

Liposarcomas usually originate in the lower extremities and retro peritoneum, whereas liposarcomas in the head and neck

\footnotetext{
Manuscript accepted for publication April 4, 2011

${ }^{a}$ Department of Otolaryngology, Gifu University Graduate School of Medicine, Japan

${ }^{b}$ Department of Tumor Pathology, Gifu University Graduate School of Medicine, Japan

${ }^{c}$ Department of Radiology, Gifu University Graduate School of Medicine, Japan

${ }^{\mathrm{d}}$ Corresponding author: Mitsuhiro Aoki, Department of Otolaryngology, Gifu University Graduate School of Medicine, 1-1 Yanagido, Gifu city, 501-1194, Japan. Email: aoki@gifu-u.ac.jp
}

doi: $10.4021 / \mathrm{jmc} 208 \mathrm{w}$ region are rare and account for only about $5 \%$ of all liposarcomas because of the relative lack in adipose tissue in these areas [1]. Primary liposarcoma in the pyriform sinus of the hypopharynx is extremely rare, with only 14 cases reported to date [2-11]. There is a distinct male preponderance and only one female case aged 90 years old has been reported. It has been previously reported that the rate of recurrence in liposarcoma of the hypopharynx is relatively high. This report presents a pregnant case of a patient with liposarcoma in the pyriform sinus of hypopharynx and also discusses the treatment options by reviewing the previous studies of hypopharyngeal liposarcoma.

\section{Case Report}

A 38-year-old female presented with dysphagia and throat discomfort at 3 months of pregnancy. She had experienced similar symptoms 10 years earlier, and lipoma-like tumor involving the pyriform sinus was removed by the intraoral approach using a direct suspension laryngomicroscope under general anesthesia twice for 2 years. The pathological diagnosis was lipoma. Three years later, the recurrent tumor mass was removed by a lateral pharyngotomy. The pathology was diagnosed as a well-differentiated liposarcoma. She

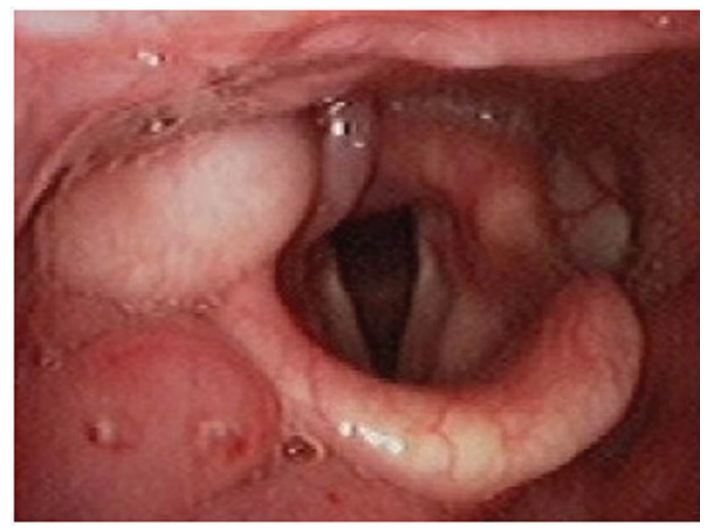

Figure 1. The endoscopy of the hypopharynx revealed a smooth tumor involving the pyriform sinus and aryepiglottic folds. 


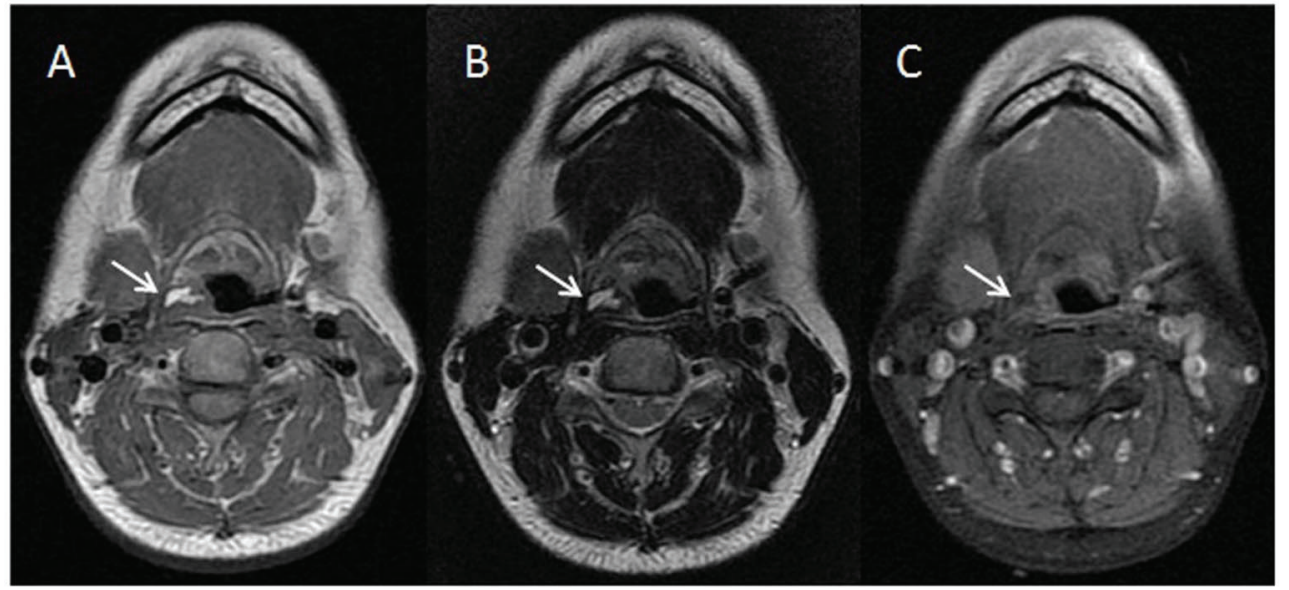

Figure 2. MRI showed a moderately heterogeneous mass spreading submucosally around the right pharynx and pyriform sinus. (A) T1 weighted image, (B) T2 weighted image, (C) fat suppression of the T1 weighted image. The white arrows show the tumor mass.

therefore underwent an additional systemic examination for metastases, however no regional and no distance metastasis were found.

Recent physical examination revealed a smooth tumor involving the pyriform sinus and aryepiglottic folds (Fig. 1). MRI showed a moderately heterogeneous mass spreading submucosally around the right pharynx and pyriform sinus with signal intensity equal to the subcutaneous fat in a T1 weighted image, and high intensity in a T2 weighted image. The mass showed low signal intensity with fat suppression of the T1 weighted image (Fig. 2).

In order to minimize a risk to the patient and the fetus, the tumor was removed by the intraoral approach using direct suspension with microlaryngoscopy and a HolmiumYAG laser under general anesthesia at 6 months of pregnancy. The size was $30 \mathrm{~mm}$ x $15 \mathrm{~mm} \times 10 \mathrm{~mm}$. Although she complained of slight dysphagia and hoarseness, oral feeding was resumed the day after the operation. She has had an un- eventful post-operative course and delivered a healthy female child without any trouble 5 months after the operation.

The histopathological findings showed that the tumor was submucosal, unencapsulated and composed of lobules of fat traversed by varying amounts of fibrous tissue-like spindle cells (Fig. 3A). Fat cells and spindle cells with enlarged and atypical nuclei were observed in the lobules (Fig. 3B). The lesion was therefore classified as well-differentiated liposarcoma.

\section{Discussion}

The presented case is the second female case with liposarcoma of hypopharynx in 15 case studies reported to date (Table 1). The liposarcoma of hypopharynx thus has a significant male predominance [3, 4]. Most of cases occur in the fourth through seventh decades of life, ranging from 23
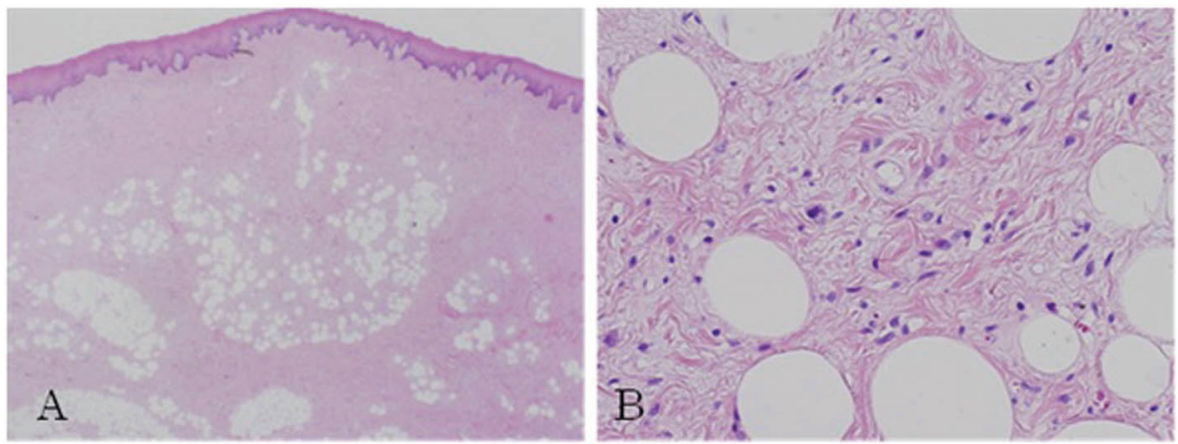

Figure 3. Pathological study. (A) Neoplastic lobules traversed by fibrous bands and composed of adipocytes of varying size (H\&E, x 40). (B) Fat cell and spindle cells with enlarged and atypical nuclei were observed in the lobules (H\&E, x 200). 


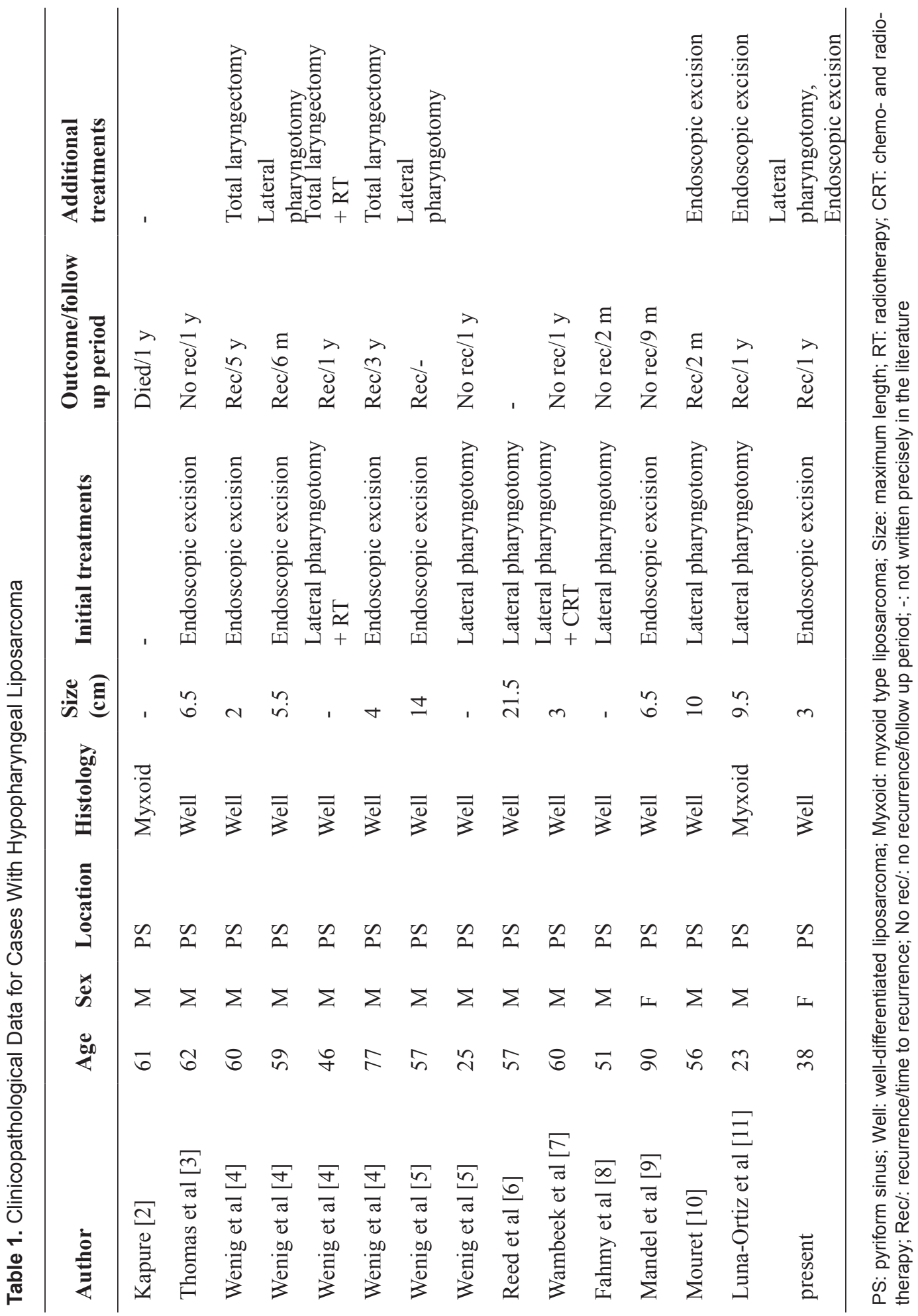


to 90 years old. The most frequent symptoms are airway obstruction, dysphagia, throat discomfort and hoarseness. The size of liposarcoma in previous reports ranged from $20 \mathrm{~mm}$ to $215 \mathrm{~mm}$.

Liposarcomas are divided into four histological types: well-differentiated type, myxoid type, round cell type and pleomorphic type [12]. The most common histological types of liposarcoma in the head and neck are the well-differentiated and the myxoid types, which account for $30 \%$ of all types each [1]. Thirteen of the 15 reported cases with liposarcoma are classified as well-differentiated type and 2 cases as myxoid type. The prognosis of the liposarcoma depends on the histological grade. The well-differentiated and the myxoid types are thought to be low grade malignancies, and the clinical courses of both types progress satisfactorily. Golledge et al. reported 5-year survival rates of $100 \%$ for well-differentiated, $73 \%$ for myxoid, $42 \%$ for pleomorphic, and $0 \%$ for round cell in 76 cases of liposarcoma of the head and neck [1].

A complete resection by wide excision is the treatment of choice for liposarcoma of the hypopharynx, as it is for liposarcoma of other regions. However, a local recurrence in cases with liposarcoma of hypopharynx has been reported in 8 cases $(61.5 \%)$ of 13 cases that included the prognosis. An endoscopic excision with laryngomicroscopy was the initial treatment in 7 cases, of which 5 cases had additional treatments for local recurrence; 3 cases by a lateral pharyngotomy and 2 cases by a total laryngectomy. A lateral pharyngotomy was the initial treatment in 6 cases, of which 3 cases received additional treatment for local recurrences. Two cases were treated by an endoscopic excision with laryngomicroscopy and one case was treated with a total laryngectomy and postoperative radiotherapy. One case was initially treated by a lateral pharyngotomy and chemo-radiation therapy. The rate of recurrence in liposarcoma of the hypopharynx is thus relatively high.

The well-differentiated type and myxoid type liposarcoma frequently recur, however they rarely show lymph node or distant metastasis [5]. Therefore, a routine neck dissection is not indicated. One fatal case with a myxoid type liposarcoma in the head and neck was reported [2], and Weiss et al. reported that the well-differentiated type of liposarcoma occasionally de-differentiates in extremities and retroperitoneal region [13]. Postoperative radiotherapy has been shown to be efficient for the myxoid type of liposarcoma [14]. However, a study recommends avoiding radiotherapy since the radiotherapy may induce de-differentiation although the chance is rare in well-differentiated liposarcoma [15]. Chemotherapeutic intervention has been performed for liposarcoma; however there is no established chemotherapy for liposarcoma [16].

The current case was treated with suspension microlaryngoscopy and the transoral approach using a HolmiumYAG laser, which is minimally invasive in comparison to a lateral pharyngotomy. The advantage of the transoral approach by laser is that oral intake can be resumed the day after the laser surgery and a tracheotomy is unnecessary in most cases. The post-operative advantage is crucial for pregnant women and aged patients. However, the disadvantage of the laser surgery is the difficulty in adequately evaluating the surgical margins via the oral approach using the laser resection in some cases due to fragmentation of the tumor. The frequent recurrence may be that liposarcoma appears to have a clear capsule whereas it often undergoes microscopic infiltration into the surrounding structures. A complete function preserving excision with clear margins may therefore constitute definitively and may be a choice of the treatment, however careful follow-up of these patients at regular intervals is also important for detecting of recurrence.

\section{Conflict of Interest}

The authors declare no conflict of interest.

\section{References}

1. Golledge J, Fisher C, Rhys-Evans PH. Head and neck liposarcoma. Cancer 1995;76(6):1051-1058.

2. Kapur TR. Recurrent lipomata of the larynx and the pharynx with late malignant change. J Laryngol Otol 1968;82(8):761-768.

3. Nofal F, Thomas M. Liposarcoma in the pharynx. J Laryngol Otol 1989;103(11):1080-1082.

4. Wenig BM, Weiss SW, Gnepp DR. Laryngeal and hypopharyngeal liposarcoma. A clinicopathologic study of 10 cases with a comparison to soft-tissue counterparts. Am J Surg Pathol 1990;14(2):134-141.

5. Wenig BM, Heffner DK. Liposarcomas of the larynx and hypopharynx: a clinicopathologic study of eight new cases and a review of the literature. Laryngoscope 1995;105(7 Pt 1):747-756.

6. Reed JM, Vick EG. Hypopharyngeal liposarcoma. Otolaryngol Head Neck Surg 1996;114(3):499-500.

7. Wambeek ND, Mendelson RM. Liposarcoma of the hypopharynx. Australas Radiol 1996;40(2):165-168.

8. Fahmy FF, Osborne J, Khalil HS, Rodgers B. Well-differentiated liposarcoma of the hypopharynx. J Laryngol Otol 1998;112(9):880-882.

9. Mandell DL, Brandwein MS, Woo P, Som PM, Biller HF, Urken ML. Upper aerodigestive tract liposarcoma: report on four cases and literature review. Laryngoscope 1999;109(8):1245-1252.

10. Mouret P. Liposarcoma of the hypopharynx. A case report and review of the literature. Rev Laryngol Otol Rhinol (Bord) 1999;120(1):39-42.

11. Luna-Ortiz K, Campos-Ramos E, Carmona-Luna T, 
Mohar-Betancourt A, Ferrari-Carballo T. Laser resection of liposarcoma of the hypopharynx. Med Oral Patol Oral Cir Bucal 2009;14(5):E252-256.

12. Weiss SW. Lipomatous tumors. Monogr Pathol 1996;38:207-239.

13. Weiss SW, Rao VK. Well-differentiated liposarcoma (atypical lipoma) of deep soft tissue of the extremities, retroperitoneum, and miscellaneous sites. A follow-up study of 92 cases with analysis of the incidence of "dedifferentiation”. Am J Surg Pathol 1992;16(11):10511058 .
14. Zagars GK, Goswitz MS, Pollack A. Liposarcoma: outcome and prognostic factors following conservation surgery and radiation therapy. Int J Radiat Oncol Biol Phys 1996;36(2):311-319.

15. Sommerville SM, Patton JT, Luscombe JC, Mangham DC, Grimer RJ. Clinical outcomes of deep atypical lipomas (well-differentiated lipoma-like liposarcomas) of the extremities. ANZ J Surg 2005;75(9):803-806.

16. Patel SR, Burgess MA, Plager C, Papadopoulos NE, Linke KA, Benjamin RS. Myxoid liposarcoma. Experience with chemotherapy. Cancer 1994;74(4):1265-1269. 